\title{
MEMS-ENABLED RETARDING POTENTIAL ANALYZERS FOR HYPERSONIC IN-FLIGHT PLASMA DIAGNOSTICS
}

\author{
E.V. Heubel, A.I. Akinwande, and L.F. Velásquez-Garcia \\ Massachusetts Institute of Technology, Cambridge, Massachusetts, USA
}

\begin{abstract}
We report the design, fabrication, and preliminary characterization of a microfabricated retarding potential analyzer (RPA) that ensures unprecedented grid alignment accuracy. Through refined manufacturing methods, improved tolerances upon assembly serve to increase the signal to noise ratio (SNR). Furthermore, microfabrication permits smaller features overcoming sensor limitations previously barring RPAs from use in very dense plasmas such as those during reentry. Preliminary results show more than a twofold increase in signal strength compared to conventional RPAs. Finally, a new batch-fabricated RPA design that uses MEMS springs for grid assembly is demonstrated to help drive down cost while improving device reliability.
\end{abstract}

\section{INTRODUCTION}

When a vehicle travels at hypersonic speed in earth's atmosphere, the heat generated through shock waves at leading edges is so intense that gas molecules surrounding the craft dissociate and ionize, generating a quasi-neutral plasma that envelops the spacecraft. These energetic plasma ions collide with the vehicle surface causing material ablation, which generates more ions while damaging and heating the craft. Diagnostic sensors can measure this harsh environment, identify exterior conditions, and while monitoring reentry can be used to help maintain adequate levels through methods such as plasma quenching (e.g. Gemini 3) [1]. Close monitoring of space missions at every step of the process helps ensure crew safety and alert ground control of serious malfunctions.

Plasmas are electrically conductive and as a result, the plasma sheath can block radio frequency communication through signal attenuation. This principle is the cause for the notorious radio blackout first experienced during the Apollo missions. The Space Shuttle and the geocentric Tracking and Data Relay Satellite System (TDRSS) worked around this problem by breaking the sheath aft of the reentry spacecraft and relaying signals back to earth through a complex satellite system $[1,2]$. However, the Shuttle is now being retired after decades of faithful service, leaving the Soyuz capsule as the sole crew return vehicle.

Therefore, in order to regain contact between mission crewmembers, identifying plasma constituents and using adaptive signaling methods is imperative. Yet strict weight requirement for space based missions, and the dense plasmas encountered at high speeds in the suborbital atmosphere, place stringent constrains on the sensor. Thus, we propose the use of microelectromechanical system (MEMS) manufacturing techniques for creating resilient, lightweight, batch-fabricated retarding potential analyzers (RPAs). RPAs measure the energy distribution of plasma ion species directly as a means of determining how harshly the sheath is impacting the craft's exterior surface. Through miniaturization, such devices may also be used in large arrays to form a highresolution sensory skin for the vehicle it equips, detecting both time- and space-resolved conditions.

\section{THEORY OF OPERATION}

An RPA is an electrostatic device that behaves in a manner analogous to a filter. Ions of sufficient energy surpass the established potential hill and penetrate the sensor to a collector plate where their flux is detected as current. Figure 1 shows a schematic of the principle of operation. When plasma of potential $V_{p}$ encounters a conductive surface, it shields itself with a sheath of a thickness proportional to the plasma Debye length. To minimize probe perturbation on the plasma, the first grid of the RPA is usually kept electrically floating to appear as any other conducting boundary surface. The induced potential due to the presence of the plasma is the floating potential, $V_{\mathrm{f}}$.

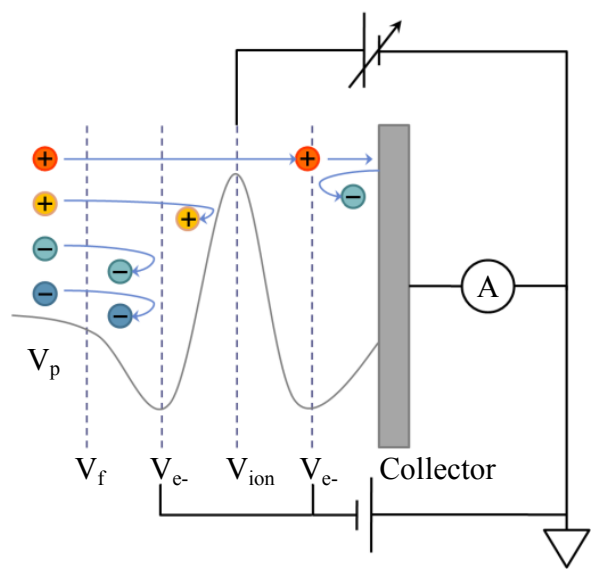

Figure 1: Schematic depicting typical RPA structure and operation

Plasma particles penetrate this self-shielding potential and enter the RPA, where the first negatively charged grid, $\mathrm{V}_{\mathrm{e}-\text {, }}$ repels incoming electrons. The voltage on the ion retarding grid, $\mathrm{V}_{\text {ion }}$, progressively shields more energetic ions as it is swept from a low potential to a high potential. Lastly, the ions make their way to the collector plate through a final negatively charged grid meant to prevent secondary electron emission or photonic emission, which would give a faulty current information [3].

The measured current is representative of the cumulative ion energy distribution. By differentiating this signal with respect to the ion retarding voltage, the transition from complete ion transmission to repulsion becomes the ion voltage distribution through the following equation

$$
-\frac{d I}{d V}=\frac{q_{i}^{2} e^{2} n_{i} A_{c}}{m_{i}} f(V)
$$

with $q_{i}$ as the ion charge state, $n_{i}$ the ion number density, $m_{i}$ ion mass, and $e$ is the elementary charge. The dynamics of RPAs have been extensively studied, yet many relationships established between the signal and the physics at play within the device are empirical in nature. This is due to concerns such as the proper representation of the RPA open area, $A_{c}$. As a result, distributions can be obtained, but their peaks are often normalized to some maximum value, not necessarily representative of the actual ion density.

$$
-\frac{d I}{d V} \sim f(V)=f\left(\frac{E_{i}}{q_{i}}\right)
$$

For a constant charge, then, assuming all atoms are singly ionized, $-d I / d V$ is directly proportional to the energy distribution [4]. 


\section{SENSOR DESIGN}

The most stringent design requirement of the RPA is Debye length, which is reduced with increasing plasma density and decreasing plasma temperature. Through the following relationship

$$
\lambda_{D}=\sqrt{\frac{\varepsilon_{0} k_{B} T_{e}}{n_{e} e^{2}}}
$$

where $\varepsilon_{0}$ is the permittivity of free space, $k_{B}$ is Boltzmann's constant, $T_{e}$ is the electron temperature, and $n_{e}$ is the electron density. To effectively shield plasma, grid openings have to be narrower than two times this critical dimension; otherwise the sheath is broken and particles may flow unobstructed to the next grid in the sensor. Hypersonic reentry experiments carried out in the late 1960s provide a measure of the plasma electron temperature and density in the sheath formed around blunt bodies [5]. The Debye lengths calculated from these parameters (Eq. 2) provide insight into the characteristic plasmas encountered (Figure 2).

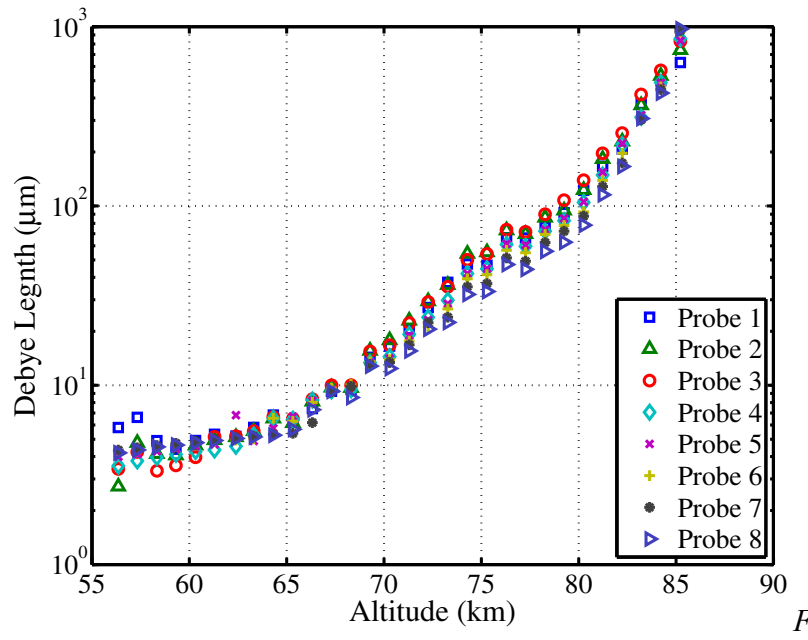

ure 2: Debye length as a function of Altitude from RAM-CII reentry experiments [5].

From Figure 2 we see that during a typical reentry, the Debye length transitions from near a millimeter to only a few microns in size. Radio blackout is usually experienced between $80 \mathrm{~km}$ and $50 \mathrm{~km}$ in altitude for the Space Shuttle, and $85 \mathrm{~km}$ to $25 \mathrm{~km}$ for the RAM-C experiments [1]. At the onset of communication loss, the Debye length is then around $100 \mu \mathrm{m}$, and will be less than $3 \mu \mathrm{m}$ at the end of the blackout period. Grids that are perforated through photochemical etching, or woven wire meshes, are typically used to establish a potential barrier and have apertures of a few hundred microns.

Limitations of present conventional manufacturing methods do not permit effective plasma shielding for these high densities without creative relaxation of the constraints. By utilizing multiple attenuating floating grids or a low transmission micro-channel plate, RPA transparency can be reduced, effectively decreasing the plasma density within the sensor by the time the first electron repelling electrode is reached [6, 7]. However, this method inherently reduces the achievable signal to noise ratio (SNR) by diminishing the flux of ions to the collector. Additionally, these materials do not permit ease of alignment of subsequent grids to better channel the remaining ions to the collector.

Through MEMS processing, micro-scale features on the order of $100 \mu \mathrm{m}$ or less, can be precisely incorporated into a device that ensures high ion transmission by enforcing optical alignment. The other limiting factor in an RPA is the inter-electrode spacing. If the gap between the electron repelling grid and ion retarding grid is too large, space-charge effects can dominate the dynamics of the RPA, i.e., a stronger than prescribed field thus established causes erroneous ion energy distributions to be measured. The acceptable value for this spacing (as derived from equating the Bohm flux to the Child-Langmuir flux) is to be less than four Debye lengths [710]. A brief review of RPAs previously built reveals the present limitations in device dimensions (Table 1).

Table 1: Overview of previous RPA characteristic dimensions. ${ }^{*}$ Note this sensor is an aligned single orifice RPA.

\begin{tabular}{|c|c|c|c|}
\hline Reference & Grid opening $(\boldsymbol{\mu m})$ & Gap $(\boldsymbol{\mu m})$ & Material \\
\hline \hline$[9]^{*}$ & 200 & 457 & Molybdenum \\
\hline$[10]$ & 140 & 500 & Stainless \\
\hline$[11]$ & 279 & 1727 & Copper \\
\hline$[12]$ & 300 & 1700 & Copper \\
\hline This work & 100 & 300 & Tungsten \\
\hline
\end{tabular}

A spacing of less than $500 \mu \mathrm{m}$ between grids is deemed difficult to maintain [8], possibly due to the fact that relatively thin meshes may short as a result of deflection through electrostatic forces when brought into proximity, as well as through thermal expansion. By using a thicker substrate, we reduce the risk of deflection, while at the same time mitigating the cupping observed in the field established by thinner electrodes [7].

As a first-generation RPA, we propose a hybrid design that incorporates micromachined grids with indexing features for alignment (Figure 3). Aspect ratios of 10:1 have been demonstrated in silicon using deep reactive ion etching (DRIE). By using $700 \mu \mathrm{m}$ thick silicon wafers, we were able to create apertures of merely $100 \mu \mathrm{m}$ in diameter. Alignment marks also served as recesses to establish an inter-electrode spacing of $300 \mu \mathrm{m}$ in conjunction with alumina washer. These dimensions are narrower than previously reported RPAs, and MEMS fabrication limitations have yet to be reached (Table 1). Additionally, in utilizing such a thick electrode material, the need for multiple ion retarding grids is averted [9]. With a conventionally machined stainless steel housing, precise assembly is achieved to a few tens of microns. The sensor is kept modular by making electrical contact through pogo pins, eliminating the need to weld wire contacts to each grid. Making use of the $60^{\circ}$ rotational symmetry of the hexagonally packed grid openings, the same pattern can be used for all four grid layers; only the collector varies in shape.

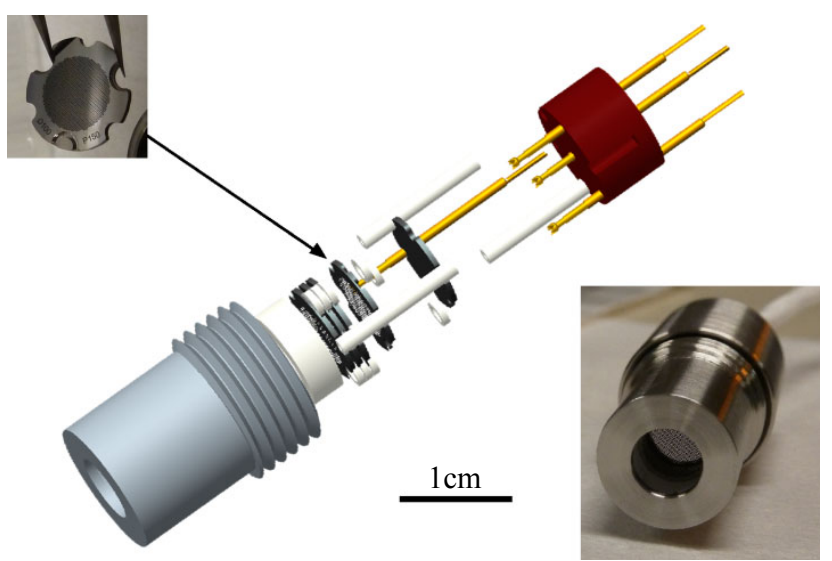

Figure 3: Assembled RPA (bottom right), exploded view of the hybrid-RPA benchmarking sensor (center) and detail of micromachined grid (left). Scale bar common to all three images. 


\section{FABRICATION}

Silicon was selected as a base material for the electrodes due to well-established processing methods, as well as its excellent mechanical performance and resilience. Two masks were used in the manufacturing of the grids to define alignment marks, spacing recesses, and apertures. First, alignment marks and recesses are defined with apertures in $10 \mu \mathrm{m}$ thick photoresist (PR) through contact photolithography and etched through DRIE (Figure $4 a, b$ ). Next, a $4 \mu \mathrm{m}$ hard oxide mask is deposited using plasma enhanced chemical vapor deposition (PECVD) and patterned with apertures and the grid boundary in $10 \mu \mathrm{m}$ PR $(4 \mathrm{c}, \mathrm{d})$. The oxide is removed using reactive ion etching (RIE), and DRIE ensues to finalize the grid pattern $(4 \mathrm{e}, \mathrm{f})$. Finally the PR and oxide are removed, and the grids are coated with tungsten $(\mathrm{W})$ by means of sputtering $(4 \mathrm{~g})$.

a)

b)

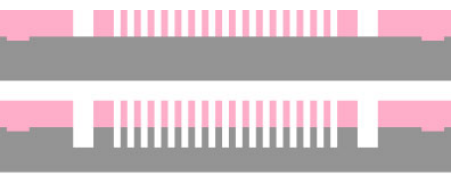

c)

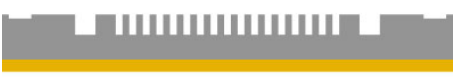

d)

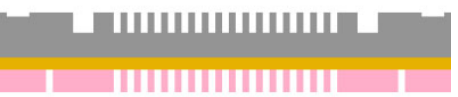

e)

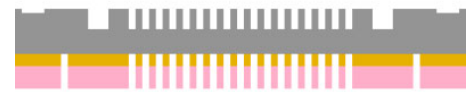

f)

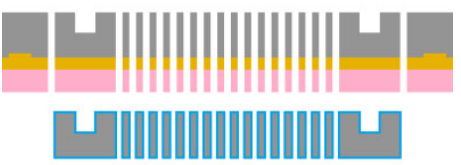

$\mathrm{Si}$

$\mathrm{Ox}$

PR

W

Figure 4: Grid process flow, a) recesses alignment marks and grid apertures defined, b) first DRIE, c) PECVD hard oxide mask deposited, d) aperture and grid edge patterned, e) hard mask RIE, f) second DRIE for release, g) W coating sputtered.

The sensor housing is milled from 316 stainless steel to a thousandth of an inch precision. Alumina rails fit into countersunk features in the main bore of the steel housing. These ensure the alignment of the $\mathrm{W}$-coated grids. The electrode stack is insulated from the device housing by means of an alumina washer and the guide rails. The small washers in Figure 3 are inserted into the DRIE grid recesses and establish an electrode spacing of $\sim 300 \mu \mathrm{m}$.

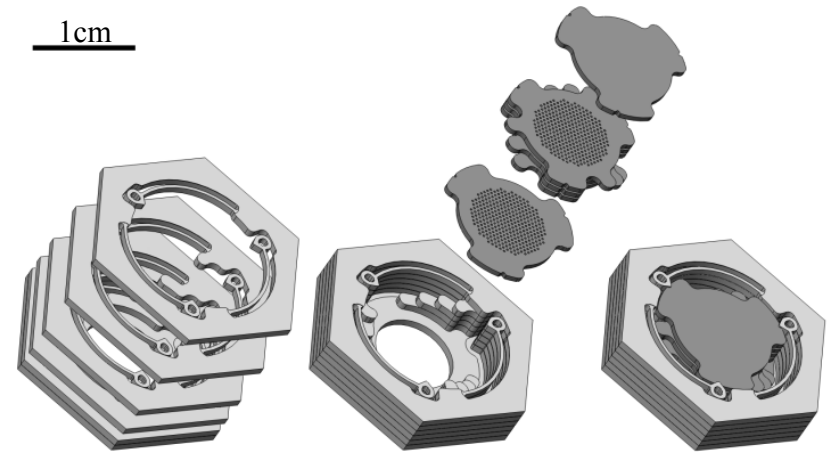

Figure 5: Exploded view of a MEMS-RPA showing housing layers prior to bonding (light), and electrode stack to be housed (dark).
The second-generation RPA will further refine grid alignment by batch microfabricating the device housing out of a silicon wafer stack. Grids will be held in place by means of curved silicon springs, and dimensional tolerances will improve by an order of magnitude to approximately one micron precision. The MEMSRPA concept is shown in Figure 5, where the retaining springs also serve as alignment enforcers. The feasibility of such mechanical assembly using DRIE silicon was demonstrated with a test structure consisting of the first three housing layers (Figure 6).

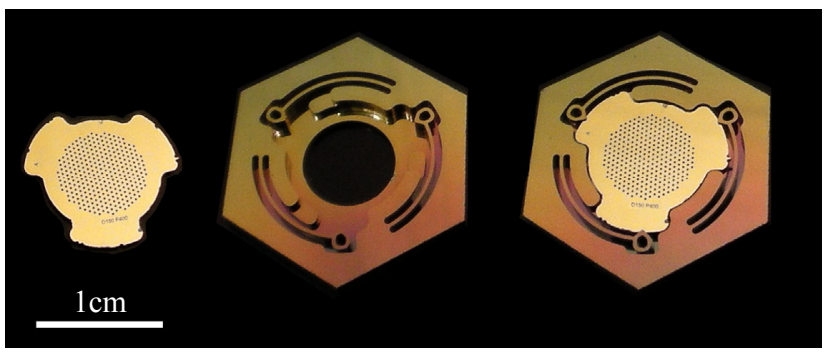

Figure 6: Grid (left), first three layers of the MEMS-RPA housing (center), and assembled grid held by retaining springs (right).

\section{DEVICE TESTING}

Preliminary tests have been carried out with the hybrid-RPA to characterize the operation of the device. The test chamber consists of an Ardara Technologies (Ardara, PA) Slim-Line ${ }^{\mathrm{TM}}$ ionizer mounted in line of sight with the RPA under test downstream of the ion beam. The thermionic ion source is operated at pressures of around $3 \times 10^{-5}$ Torr. The filament generates electrons of $-50 \mathrm{eV}$ energy using an emission current of $0.2 \mathrm{~mA}$. For simplicity, air is used as the ionized gas, and the ion energy is controlled by a potential assigned to a gold cage surrounding their nascent origin.

Since we are using a plasma-less ion source, the floating grid is removed from the RPA. Due to the absence of electrons from the flow, the potential established on a floating grid would effectively repel all impinging ions. Thus, the first grid is the electron repelling electrode, which in our experiments - along with the secondary electron suppression grid - is held at a $-10 \mathrm{~V}$ bias. The ion retarding grid is swept from $-15 \mathrm{~V}$ to $15 \mathrm{~V}$, and currents from these two biasing potentials and the collector plate (at ground) are synchronously recorded. Three Keithley 237 source measure units (SMUs) measure the charge flux to five digits of precision approximately every $500 \mathrm{~ms}$.

\section{EXPERIMENTAL RESULTS}

Data were first collected operating the chamber with a $10 \mathrm{~V}$ ion region. In these conditions, the expected particle energy for singly-charge ions is $10 \mathrm{eV}$. The first recorded trace with the hybrid-RPA shows a peak that is nearly $5 \mathrm{~V}$ lower. Subsequent tests maintained a $4-6 \mathrm{~V}$ discrepancy between the anticipated location of the peak distribution and that measured with our device. Using our modular hybrid design with stainless steel mesh grids instead of our precision grids; a conventional RPA is thus created for benchmarking purposes. This more standard sensor with apertures of $152 \mu \mathrm{m}$ showed a similar shift in peak, while signal strength was cut to less than half (Figure 7).

Comparing the data from the RPA with micromachined grids to an RPA with standard stainless steel mesh, preliminary results show improved signal strength with mechanically enforced optical transparency. A narrower, more prominent peak is observed using our hybrid-RPA. In order to further diagnose the shift in energy, various ion region voltages were tested (Figure 8). Our device was 
shown to reliably follow this increasing energy, and the offset at this time is considered a calibration issue of the instrument. However, we captured an anomaly with the hybrid-RPA data, in the form of a non-physical negative distribution. The apparent negative distribution is believed to result from ion beam focusing and defocusing effects within the device. To better understand this signal oddity, the electron suppression grids were disconnected from one another and measured individually using a fourth Keithley SMU. The second electron repelling grid should be expendable because ion energies presently used are too low (well below $1 \mathrm{keV}$ ) to induce secondary emission [10]. The prognosis is that this grid is not only unnecessary but in fact reduces the ion flux to the collector plate. By combining the collector and secondary electron repelling grid currents (as two collectors), the reported distribution is once again positive (not shown).

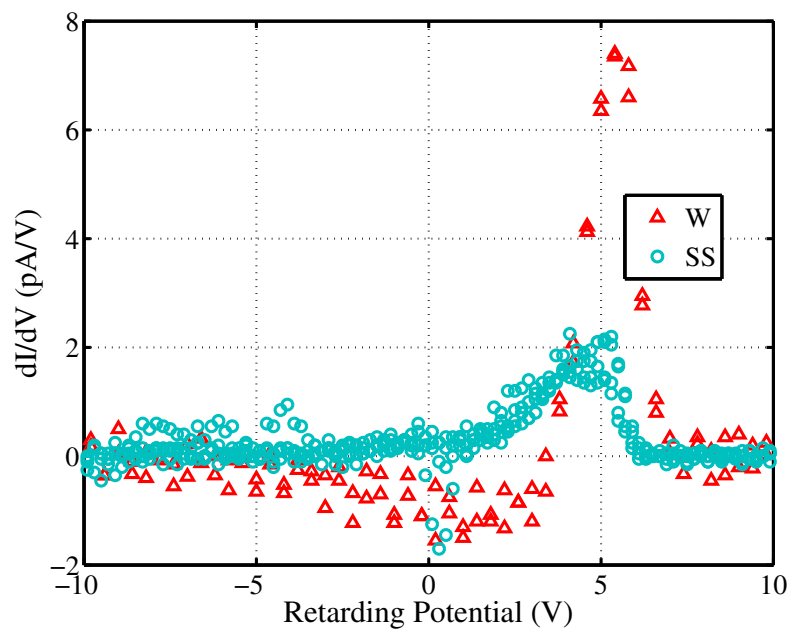

Figure 7: Measured energy distribution comparing tungstencoated aligned micromachined grids (W) to conventional unaligned stainless mesh (SS). A narrow distribution and more intense peak is obtained with the MEMS design.

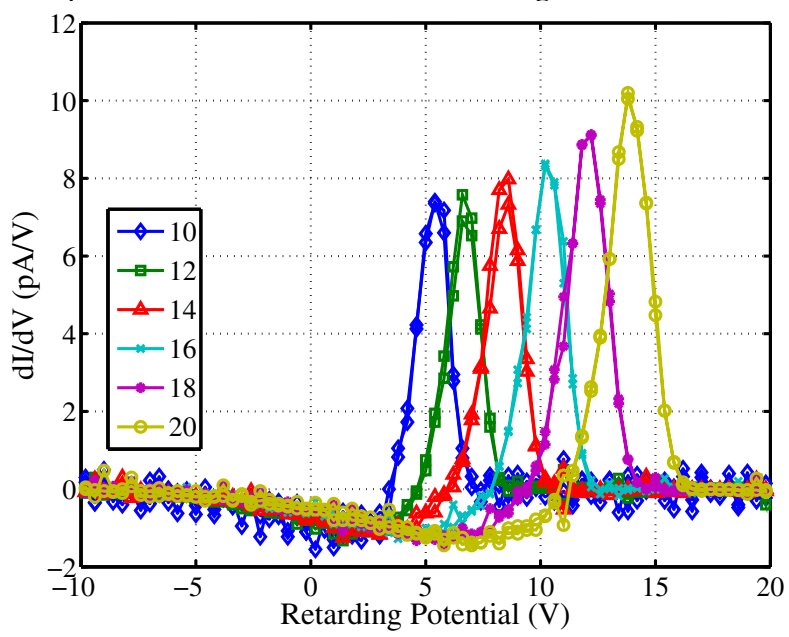

Figure 8: Collected energy distributions using the hybrid-RPA with tungsten-coated grids. Traces demonstrate that the sensor detects the prescribed increase in ion energy.

\section{CONCLUSION}

We preliminarily demonstrated a novel RPA using MEMS machining techniques to enforce grid alignment. With increased optical transparency, signal strength was improved by a factor of more than two over conventional RPAs. By incorporating microscale features and improving construction tolerances, devices can be made compatible with denser plasmas while maintaining large SNR. Thicker electrode material improve the longevity of RPAs in harsh environments, while reducing the effect of electric field cupping, removing the need for multiple ion retarding electrodes. A second-generation RPA is proposed and we demonstrated using a test structure the feasibility of assembling grids using MEMS deflection springs.

\section{ACKNOWLEDGEMENTS}

The microfabrication was conducted at MIT's Microsystems Technology Laboratories. This work was supported by NASA under Award No. NNC08CA58C (program managers R. Manning and T. Wallett). Any opinions, findings, and conclusions or recommendations expressed in this publication are those of the author(s) and do not necessarily reflect the views of NASA and therefore, no official endorsement of NASA should be inferred.

\section{REFERENCES}

[1] R. A. Hartunian, G. E. Stewart, S. D. Fergason, T. J. Curtiss, and R. W. Seibold, "Causes and Mitigation of Radio Frequency (RF) Blackout During Reentry of Reusable Launch Vehicles," The Aerospace Corporation, (2007).

[2] K. M. Lemmer, A. D. Gallimore, and T. B. Smith, "Using a helicon source to simulate atmospheric re-entry plasma densities and temperatures in a laboratory setting," Plasma Sources Science and Technology, 18, (2009), pp. 1 - 8.

[3] M. C. Kelley, The Earth's Ionosphere: Plasma Physics and Electrodynamics, Academic Press Inc., Boston, MA, $2^{\text {nd }}$ edition, 2009.

[4] L. B. King, "Transport-Property and Mass Spectral Measurements in the Plasma Exhaust Plume of a Hall-Effect Space Propulsion System," $\mathrm{PhD}$ thesis, University of Michigan, 1998.

[5] W. L. Jones Jr. and A. E. Cross, "Electrostatic-probe measurements of plasma parameters for two reentry flight experiments at 25000 feet per second," NASA Tech. Report NASA, Langley Research Center, Hampton, VA, (1972).

[6] K. M. Lemmer, A. D. Gallimore, T. B. Smith, and D. R. Austin, "Review of two retarding potential analyzers for use in high density helicon plasma," $30^{\text {th }}$ International Electric Propulsion Conference, Florence, Italy, (2007).

[7] J. M. Partridge, "Development and Implementation of Diagnostics for Unsteady Small-Scale Plasma Plumes," PhD thesis, Worcester Polytechnic Institute, September 2008.

[8] I. H. Hutchinson, Principles of Plasma Diagnostics, Cambridge University Press, New York, NY, $2^{\text {nd }}$ edition, 2002.

[9] C. M. Marrese, N. Majumdar, J. M. Haas, G. Williams, L. B. King, and A. D. Gallimore, "Development of a Single-orifice Retarding Potential Analyzer for Hall Thruster Plume Characterization," Proceedings of the $25^{\text {th }}$ International Electric Propulsion Conference, Cleveland, OH, (1997).

[10] Y. Azziz, "Experimental and Theoretical Characterization of a Hall Thruster Plume," PhD thesis, Massachusetts Institute of Technology, June 2007.

[11] B. E. Beal and A. D. Gallimore, "Energy analysis of a Hall thruster cluster," $28^{\text {th }}$ International Electric Propulsion Conference, Toulouse, France, (2003).

[12] R. R. Hofer, J. M. Haas, and A. D. Gallimore, "Ion voltage diagnostics in the far-field plume of a high-specific impulse Hall thruster," $39^{\text {th }}$ AIAA/ASME/SAE/ASEE Joint Propulsion Conference, Huntsville, AL, (2003). 